\title{
ECMO after cardiac surgery: a single center study on survival and optimizing outcomes
}

\author{
Jennifer M. Brewer ${ }^{1 *} \mathbb{D}$, Anthony Tran ${ }^{1}$, Jielin Yu ${ }^{1}$, M. Irfan Ali ${ }^{1}$, Constantine M. Poulos ${ }^{1}$, Jonathan Gates ${ }^{2}$, \\ Jason Gluck ${ }^{3}$ and David Underhill ${ }^{4}$
}

\begin{abstract}
Background: The study purpose is to examine survival prognostic and extracorporeal membrane oxygenation (ECMO) application outcomes at our tertiary care center.

Methods: This is a retrospective analysis, January 2014 to September 2019. We analyzed 60 patients who underwent cardiac surgery and required peri-operative ECMO. All inpatients with demographic and intervention data was examined. 52 patients (86.6\%) had refractory cardiogenic shock, 7 patients (11.6\%) had pulmonary insufficiency, and 1 patient (1.6\%) had hemorrhagic shock, all patients required either venous-arterial (VA) ( $n=53,88.3 \%)$, venousvenous (VV) $(n=5,8.3 \%)$ or venous-arterial-venous (VAV) $(n=2,3.3 \%)$ ECMO for hemodynamic support. ECMO parameters were analyzed and common postoperative complications were examined in the setting of survival with comorbidities.
\end{abstract}

Results: In-hospital mortality was $60.7 \%(n=37)$. Patients who survived were younger $(52 \pm 3.3$ vs $66 \pm 1.5, p<0.001)$ with longer hospital stays ( $35 \pm 4.0$ vs $20 \pm 1.5, p<0.03)$. Survivors required fewer blood products $(13 \pm 2.3$ vs $25 \pm 2.3$, $p=0.02)$ with a net negative fluid balance $(-3.5 \pm 1.6$ vs $3.4 \pm 1.6, p=0.01)$. Cardiac re-operations worsened survival.

Conclusion: $\mathrm{ECMO}$ is a viable rescue strategy for cardiac surgery patients with a $40 \%$ survival to discharge rate. Careful attention to volume management and blood transfusion are important markers for potential survival.

Keywords: Cardio-thoracic surgery, ECMO, Cardiac transplantation, Critical care, LVAD, Cardiac surgery

\section{Background}

Myocardial dysfunction after cardiac surgical intervention occurs in about $3-8 \%$ of patients $[1,2]$. Patients are typically separated from cardiopulmonary bypass with inotropes/vasopressors or intra-aortic balloon counterpulsation [3-5]. In the event of refractory cardiac and/ or pulmonary dysfunction additional mechanical circulatory support may be required. Extracorporeal membrane oxygenation (ECMO) is a bridging mechanical circulatory support with promising results seen in a multitude of post cardiotomy procedures with poor residual cardiac

\footnotetext{
*Correspondence: Jenbrewer830@gmail.com

${ }^{1}$ Department of General Surgery, University of Connecticut, 263 Farmington Ave, Farmington, CT 06030, USA
}

Full list of author information is available at the end of the article function [1, 6-9]. Prognostic factors previously identified include: preventing left ventricular overloading, pulmonary edema, lung injury, and myocardial damage. Additional prognostic indicators are low oxygen pressure and low oxygen saturation of ECMO tubing, advanced age, pre-operative co-morbidities, the type of surgical procedure, and high blood product requirement [10-17]. We analyzed 60 patients who underwent cardiac surgery and required peri-operative ECMO support from January 2014 to September 2019. The purpose of this study is to delineate prognostic factors of survival, discuss the outcomes, and elaborate on the application of ECMO at a tertiary care center.

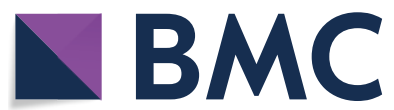

(c) The Author(s) 2021. Open Access This article is licensed under a Creative Commons Attribution 4.0 International License, which permits use, sharing, adaptation, distribution and reproduction in any medium or format, as long as you give appropriate credit to the original author(s) and the source, provide a link to the Creative Commons licence, and indicate if changes were made. The images or other third party material in this article are included in the article's Creative Commons licence, unless indicated otherwise in a credit line to the material. If material is not included in the article's Creative Commons licence and your intended use is not permitted by statutory regulation or exceeds the permitted use, you will need to obtain permission directly from the copyright holder. To view a copy of this licence, visit http://creativecommons.org/licenses/by/4.0/. The Creative Commons Public Domain Dedication waiver (http://creativeco mmons.org/publicdomain/zero/1.0/) applies to the data made available in this article, unless otherwise stated in a credit line to the data. 


\section{Methods}

From January 2014 to September 2019, we performed over 6000 cardiac surgeries and over 250 ECMO implants of which 60 were placed in cardiotomy patients. The patients requiring ECMO support following primary cardiac surgery underwent the following procedures: coronary artery bypass grafting (CABG), valvular surgery, CABG with concomitant valvular surgery, heart transplantation, aortic root repair, left ventricular assistant device (LVAD) implantation, descending aortic repair, and other procedures. Nine cases were implanted at institutions outside our network and brought back to our hub hospital. Patients were placed on venous-arterial (VA), venous-venous $(\mathrm{VV})$, or veno-arterio-venous (VAV) ECMO. Indications for placing a patient on ECMO included cardiogenic shock, pulmonary insufficiency or hemorrhagic shock. Cardiogenic shock was defined as more than two vasoactive drugs and escalating care, or intra-aortic balloon pump (IABP) with more than one vasoactive drug and escalating care, or cardiac arrest with return of spontaneous circulation. Respiratory failure was defined as $\mathrm{pO} 2<50$ and $\mathrm{FiO} 2>60 \%$ for at least $12 \mathrm{~h}$, or $\mathrm{pH}<7.2$ despite optimal vent settings, or $\mathrm{P} / \mathrm{F}$ ratio $<80$ with high levels of PEEP. We used regional $\mathrm{SO}^{2}$ monitors to ensure that when the femoral artery was cannulated there was perfusion to that extremity from our routinely performed jump grafts. We have a specific ECMO anticoagulation protocol for post cardiac surgery patients. Heparin was reversed in the operating room $(\mathrm{OR})$ with protamine sulfate and anticoagulation was typically held for $24 \mathrm{~h}$ post-operatively. Patients were then usually anticoagulated with 500-700units/ per hour of heparin as a fixed dose and without a bolus for $6 \mathrm{~h}$ then gently uptitrated to achieve an anti-Xa level of 0.3-0.5. If cannulation occurred in the OR we performed direct surgical cannulation of the right atrium and aorta. The size of the cannula was surgeon preference. Usually for the atrium this is about a 28-32 French cannula, for the aorta this is about a 20 French cannula. We used multilevel measures to ensure that the cannulas are secured, such as pledgeted horizontal mattress sutures or purse-string sutures. At the end of the procedure, we typically close the chest and we tunnel the cannula through soft tissues via separate incisions, however as needed we left the chest open. In terms of weaning ECMO we began by weaning pressor and inotropic support. We weaned down the ECMO flow $(500 \mathrm{cpm})$ and volume as we continued to diuresis. We monitored vitals as we attempted to wean ECMO. We employed lung protection ventilation as we worked to wean ECMO. Weaning continued until patients are on minimal settings with stable vitals.

\section{Data collection}

After obtaining Hartford Hospital IRB approval (HHC2019-0189), we retrospectively reviewed prospectively collected data in our ECMO patient database and electronic health record. The primary outcome was survival to discharge. Demographics were collected to delineate age, gender, Body Mass Index (BMI), total hospital days admitted, total days on ECMO support, and ECMO type/modality. The following comorbidity data was also collected: diabetes, hypertension, coronary artery disease (CAD), history of myocardial infarction (MI), history of prior CABG, chronic obstructive pulmonary disease (COPD), asthma, congestive heart failure (CHF), peripheral vascular disease (PVD), renal disease, history of dialysis, autoimmune disease, malignancy, hyperlipidemia, pre-operative ejection fraction $(E F)$, and pulmonary hypertension. Pre-operative ejection fraction was defined as the EF before undergoing any procedure, including catheterization laboratory procedures.

Cases were classified as elective, urgent and emergent using standard Society of Thoracic Surgery definitions. Elective cases were classified as the patient's cardiac function has been stable for days or week prior to the operation, therefore, the procedure could be deferred without increased risk of compromised cardiac outcome. Urgent cases were classified as procedures required during the same hospitalization. These patients had to remain in the hospital until the surgery could take place, but the patient is able to wait for surgery until the next available operating room scheduled time. Emergent cases were classified as requiring emergency operations. These patients would have had ongoing, refractory, unrelenting cardiac compromise, with or without hemodynamic instability, which not responsive to any form of therapy. An emergency operation is one in which there should be no delay in providing operative intervention.

Perioperative and postoperative ECMO data was analyzed with respect to blood product use, overall volume status, and whether we placed ECMO at an outside hospital (ECMO-on-the-go) or in our institution. Postoperative complications included renal failure, sepsis, intracranial hemorrhage/anoxic brain injury, deep venous thromboembolism/pulmonary embolus (DVT/ $\mathrm{PE}$ ), ischemic limb, and cardiac reoperation. Renal failure was defined according to the Second International Consensus Conference of the Acute Dialysis Quality Initiative Group. Sepsis was defined as systemic inflammatory response syndrome (SIRS) with any known source. Anoxic brain injury in our study was defined as any imagining findings that demonstrated anoxic brain injury and confirmed by supporting documentation in the patient's chart by a physician. The patients 
were divided into two groups: those patients that survived to discharge and those that expired after ECMO postoperatively.

\section{Statistical analysis}

Continuous data is presented as a mean \pm standard error or deviation if normally distributed, or as a median and interquartile range (25th, 75 th) if non-normally distributed. Continuous variables were assessed with a Student's t-test when normally distributed and Wilcoxon signed rank-sum test when data is skewed. Fisher's exact test was used to assess categorical variables. Mann- $U$ Whitney test was used to assess rank sums of data not normally distributed. Significant differences were denoted by a $p$ value of $\leq 0.05$ in all statistical analyses with a $95 \%$ confidence interval. We performed a descriptive analysis focusing on clinical outcomes assessed. Statistical analysis was carried out with IBM $^{\mathrm{TM}}$ SPSS Statistics for Windows/Mac, version 26 (IBM Corporation, Armonk, NY 2019) and Graphpad Prism for Windows/Mac, version 8.31 (GraphPad Software, La Jolla California USA).

\section{Results}

A total of 60 patients were included in this study (Table 1$)$. In-hospital mortality was $60.7 \%(n=37)$ with 23 patients surviving to discharge. 52 of $60(86.6 \%)$ patients had cardiogenic shock requiring V-A ECMO (Table 2), 7 of 60 (11.6\%) had respiratory failure requiring V-V ECMO and 1 (1.6\%) patient had hemorrhagic shock and was placed on V-A ECMO (Table 2). The one patient who experienced hemorrhagic shock had a prolonged hospital course, ultimately requiring ECMO cannulation after sternal wound dehiscence led to profound bleeding. 2 patients were placed on V-A-V ECMO after being initially placed on V-V ECMO and were included in the V-V cohort, as outlined above.

43 of $60(71.6 \%)$ were placed on ECMO in the OR, 7 (11.6\%) were placed on peripheral ECMO pre-operatively in the catheterization laboratory and 10 (16.6\%) were placed on peripheral or central ECMO post-operatively in the intensive care unit (ICU).

14 of 43 (32\%) patients, survived having ECMO placed in the operating room. 5 of 7 (71.1\%) patients survived pre-operative ECMO placement in the

Table 1 Patient demographics

\begin{tabular}{|c|c|c|c|}
\hline Demographics & $\begin{array}{l}\text { Non-survivors } \\
(\mathrm{N} \text { total }=37)\end{array}$ & $\begin{array}{l}\text { Survivors } \\
(\mathrm{N} \text { total }=23)\end{array}$ & $p$ \\
\hline $\begin{array}{l}\text { Age }(y) \\
(\text { Mean } \pm \text { STD })\end{array}$ & $66 \pm 1.5$ & $52 \pm 3.3$ & $<0.001$ \\
\hline Male & 22 & 17 & 0.59 \\
\hline Female & 14 & 7 & 0.59 \\
\hline $\begin{array}{l}\text { BMI }\left(\mathrm{kg} / \mathrm{m}^{2}\right) \\
(\text { Mean } \pm \mathrm{STD})\end{array}$ & $34 \pm 1.4$ & $31 \pm 1.4$ & 0.16 \\
\hline \multicolumn{4}{|l|}{ Past medical history } \\
\hline Diabetes & 8 & 8 & 0.38 \\
\hline Hypertension & 23 & 13 & 0.30 \\
\hline Coronary artery disease & 19 & 6 & 0.037 \\
\hline Myocardial infarction & 6 & 2 & 0.46 \\
\hline Pre-op coronary artery bypass graft & 7 & 1 & 0.12 \\
\hline Chronic obstructive pulmonary disease & 5 & 0 & 0.077 \\
\hline Asthma & 5 & 3 & 1.0 \\
\hline Congestive heart failure & 14 & 8 & 0.78 \\
\hline Peripheral vascular disease & 2 & 0 & 0.51 \\
\hline Renal disease & 7 & 6 & 0.75 \\
\hline Dialysis & 3 & 2 & 1.00 \\
\hline Autoimmune disease & 6 & 0 & 0.072 \\
\hline Malignancy & 5 & 0 & 0.0768 \\
\hline Hyperlipidemia & 18 & 9 & 0.43 \\
\hline Pulmonary hypertension & 3 & 1 & 0.64 \\
\hline Smoker & 16 & 7 & 0.41 \\
\hline History of cardiac surgery & 8 & 1 & 0.13 \\
\hline
\end{tabular}

STD standard deviation, OHT open heart transplant 
Table 2 ECMO indications, timing of ECMO placement, and type of ECMO

\begin{tabular}{|c|c|c|c|}
\hline Indications for ECMO & Non-survivors $(\mathrm{N}$ total $=37$ ) & Survivors $(\mathrm{N}$ total $=23)$ & $\mathrm{p}$ \\
\hline Cardiogenic shock & 33 & 19 & 0.25 \\
\hline Pulmonary insufficiency & 3 & 4 & 0.42 \\
\hline Other (hemorrhagic shock) & 1 & 0 & 1.00 \\
\hline \multicolumn{4}{|l|}{ ECMO type } \\
\hline VA & 34 & 19 & $<0.00001$ \\
\hline W & 2 & 3 & 0.38 \\
\hline VAV & 1 & 1 & 1.00 \\
\hline \multicolumn{4}{|l|}{ Timing of ECMO placement } \\
\hline Pre-operatively (catheterization laboratory) & 2 & 5 & 0.09 \\
\hline Intra-operative (operating room) & 29 & 14 & 0.24 \\
\hline Post-operatively (ICU post-operative) & 6 & 4 & 1.00 \\
\hline
\end{tabular}

$V A$ venoarterial, $V V$ venovenous, $V A V$ Veno-arterio-venous

catheterization laboratory. 4 of 10 (40\%) patients survived ECMO placement post-operatively in the ICU (Table 2).

33 of the 43 patients who had ECMO placed in the OR were centrally cannulated. 5 of 10 patients who had ECMO placed in the ICU were cannulated centrally because their chests were already open. All catheterization lab patients had peripheral cannulation. Of the 43 patients who had ECMO placed in the OR, 35 received $\mathrm{V}$-A ECMO. 4 of 7 patients who had ECMO placed in the catheterization laboratory required V-A ECMO. 9 of 10 patients placed on ECMO in the ICU were placed on $\mathrm{V}$-A ECMO. There was no significant difference between survivors and non-survivors in time of ECMO implantation, pre-operatively (catheterization laboratory), intraoperatively or post-operatively (in the ICU). All patients who had pre-operative ECMO placed in the catherization then proceeded directly to the OR.

Overall duration of ECMO support in all patients was a median of 5 days (2-8 days). Between survivors and non-survivors, there was no difference in the number of days patients were on ECMO (survivors 4.8 days vs nonsurvivors 5.7 days, $p=0.33$ ) (Table 3 ). However, we did find that fluid balance was significantly different between survivors and non-survivors. Survivors achieved a net negative fluid balance while on ECMO $(-3.5 \mathrm{~L}$ total in survivors vs $+3.4 \mathrm{~L}$ total in non-survivors, $p=0.01$ ) (Table 3).

Of the 23 patients who survived to discharge 2 patients were discharged with an LVAD, with one of those patients ultimately receiving an Orthotropic Heart Transplant (OHT). Of the other 21 patients who survived, 6 underwent OHT, 15 recovered sufficiently for decannulation and discharge. All patients who survived were discharged either to home or a rehabilitation facility, with every patient eventually being transitioned home.
Table 3 ECMO outcomes

\begin{tabular}{llll}
\hline Outcome & $\begin{array}{l}\text { Non-survivors } \\
\text { (N total=37) }\end{array}$ & $\begin{array}{l}\text { Survivors } \\
(\mathbf{n = N} \\
\text { Total=23) }\end{array}$ & $\boldsymbol{p}$ \\
\hline Total hospital LOS (days) & $20 \pm 1.5$ & $35 \pm 4.0$ & 0.03 \\
$\begin{array}{l}\text { ECMO support (days) } \\
\text { Blood product use while }\end{array}$ & $5.7 \pm 0.73$ & $4.8 \pm 0.53$ & 0.33 \\
$\begin{array}{l}\text { On ECMO (mean units of } \\
\text { PRBCs } \pm \text { STD) }\end{array}$ & $25 \pm 2.3$ & $13 \pm 2.3$ & 0.02 \\
Bleeding events & $19(51.3 \%)$ & $8(34.7 \%)$ & 0.28 \\
Net volume status (L & $3.4 \pm 1.6$ & $(-) 3.5 \pm 1.5$ & 0.01 \\
mean \pm STD) & $20(54.0 \%$ & $7(30.4 \%)$ & 0.064 \\
Inotrope/vasopressor use & $15(83.3 \%)$ & $3(16.6 \%)$ & 0.02 \\
eCPR & $4(44.4 \%)$ & $5(55.5 \%)$ & 0.29 \\
ECMO on the go & 0 & 4 & 0.018 \\
OHT as salvage procedure & & & \\
\hline
\end{tabular}

LOS length of stay, STD standard deviation, OHT open heart transplant

9 of 60 (15\%) patients underwent ECMO-on-the-go. 5 of these $9(55.5 \%)$ patients survived. However, this was not statistically different $(\mathrm{p}=0.29)$.

\section{Demographics}

Survivors were found to be younger ( 52 years vs 66 years, $p<0.001$ ) (Table 1 ). The average age of all patients was $60 \pm 1.79$ years old. 19 of $37(51.3 \%)$ non-survivors had a past medical history of CAD. This was the only past medical history analyzed that was found to be significantly more prevalent in non-survivors than survivors $(\mathrm{p}=0.037)$. Overall, our patient's mean preoperative EF was $42 \%$, this was not significantly different between survivors and non-survivors $(\mathrm{p}=0.30)$.

22 of $60(36.6 \%)$ cases were elective, 10 of 60 (16.6\%) cases were emergent, and 28 of 60 (45\%) cases were urgent (Table 4). There was no statistical difference between survivors and non-survivors based on operation 
Table 4 Operation classification

\begin{tabular}{llll}
\hline $\begin{array}{l}\text { Operation } \\
\text { classification }\end{array}$ & $\begin{array}{l}\text { Non-Survivors (N } \\
\text { total=37) }\end{array}$ & $\begin{array}{l}\text { Survivors (N } \\
\text { total=23) }\end{array}$ & $\boldsymbol{p}$ \\
\hline Elective & 16 & 6 & 0.27 \\
Emergent & 7 & 3 & 0.73 \\
Urgent & 14 & 14 & 0.19 \\
\hline
\end{tabular}

Table 5 Primary surgery

\begin{tabular}{llll}
\hline Primary surgery & $\begin{array}{l}\text { Non-survivors (N } \\
\text { total=37) }\end{array}$ & $\begin{array}{l}\text { Survivors (n } \\
\text { total=23) }\end{array}$ & $\boldsymbol{p}$ \\
\hline CABG & 10 & 2 & 0.16 \\
Valve repair & 10 & 7 & 0.77 \\
CABG + valve repair & 4 & 2 & 1.00 \\
OHT & 2 & 2 & 0.04 \\
Aortic root repair & 5 & 2 & 0.69 \\
LVAD placement & 3 & 1 & 1.00 \\
Descending aortic repair & 0 & 2 & 0.14 \\
Less commonly performed & 2 & 6 & 0.04 \\
procedures & & &
\end{tabular}

CABG coronary artery bypass surgery, OHT open heart surgery, LVAD left ventricular assist device

classification ( $p>0.05)$. In addition, ECMO cardiopulmonary resuscitation was initiated either in the operative theater or post-operatively in the ICU. ECMO cardiopulmonary resuscitation (eCPR) had a high incidence of mortality. 12 of 15 (80\%) ECMO patients who had eCPR did not survive (Table 3).

12 of $60(20 \%)$ operative cases were isolated CABGs. Non-survivors $(n=10)$ had a mean of 2.86 vessel disease repaired whereas survivors $(n=2)$ had a mean of 3.75 vessel disease repaired. 17 of $60(28.3 \%)$ patients underwent isolated valve repair. 6 of $60(10 \%)$ patients underwent CABG and valve repair. 7 of $60(11.7 \%)$ patients underwent aortic root repair. 4 of $60(6.6 \%)$ patients had a LVAD placement. 2 of $60(3.3 \%)$ patients underwent descending aortic repair. 8 of $60(13.3 \%)$ patients underwent less commonly performed open heart procedures. 4 of $60(6.6 \%)$ patients underwent OHT as their primary surgery (Table 5).

However, in total 8 of $60(13.3 \%)$ patients underwent OHT. 4 patients had OHT as their primary procedure and required ECMO to be brought out of the OR. 2 of the patients who had OHT performed as a primary procedure did not survive. Primary OHT was the only heart surgical procedure found to be significantly different between survivors and non-survivors $(p=0.04) .4$ patients underwent OHT after being placed on ECMO as a salvage for their original cardiac surgical procedure.
These 4 patients already had a primary open-heart surgery and OHT was attempted as a salvage procedure.

\section{ECMO secondary outcomes and adverse events}

19 of 37 non-survivors (51.3\%) had a bleeding event while on ECMO. 5 of these 19 patients needed re-operation of their chest. 8 of 23 (34.7\%) survivors had a bleeding event on ECMO. 2 of these 8 patients needed re-operation of their chest for bleeding. This was not significantly different between survivors and non-survivors, but trending towards increased bleeding events in non-survivors $(p=0.28)$. Survivors needed a mean 13 units of blood, while non-survivors needed a mean of 25 units of blood, this was found to be significantly different $(p=0.02)$.

14 of $37(37.8 \%)$ non-survivors required re-operation, which was significantly more than survivors $(\mathrm{p}=0.04)$. 3 of $23(13.0 \%)$ survivors required re-operation, 2 for bleeding and 1 for sternal wound dehiscence. 20 (54.0\%) non-survivors and $5(21.7 \%)$ survivors developed renal failure requiring renal replacement therapy without significant difference between these groups $(\mathrm{p}=0.16) .18$ (48\%) non-survivors and 13 (56.5\%) survivors developed sepsis, again, not statistically different $(\mathrm{p}=0.6) .8(21.6 \%)$ non-survivors and 1 (4.34\%) survivor developed intracerebral hemorrhage/anoxic brain injury which was not significantly different $(\mathrm{p}=0.13) .1(2.7 \%)$ non-survivor and 2 (8.6\%) survivors developed DVT/PE, which was not significantly different $(\mathrm{p}=0.56)$. 4 Patients ( 3 survivors), had clotting in their ECMO circuit, of these 2 patients were not anticoagulated with heparin and occurred at a mean of 10 days. Of note, one patient had thrombosis of the circuit in the OR and did not survive.

\section{Discussion}

ECMO is a valuable therapeutic option for postcardiotomy, particularly when the underlying cause is thought to be reversible $[4-7,11-13]$. This series demonstrates a heterogenous range of cardiac procedures with a similar duration of ECMO support. Determining risk profile in those patients that survive ECMO demonstrates important findings that may suggest means to improve hospital management.

Our paper focuses on increasing survival in patients who do not survive cardiac surgery. We believe ECMO use helps stabilize these patients for eventual discharge. In our patient cohort, in-hospital mortality was $60 \%$. The literature on ECMO placement following cardiac surgery has similar mortality rates of $64-67 \%$ representing the overall tenuous underlying pathophysiology in these patients [14, 17]. Without ECMO use, we believe the $40 \%$ of survivors in our study would have died without mechanical circulatory support $[15,17]$. 
Our observed mean duration of ECMO support was 5 days, similar to that reported of 5.4-7.5 days, without significant difference for survivors and non-survivors $[14,15,17]$. Therefore, we suggest transparent discussion with families and patients regarding potential outcomes and allowing up to 8 days on ECMO before making vital decisions. One marker that stood out as a predictor of survival in our study is achieving overall net negative fluid balance $(-3.5 \mathrm{~L}$ vs $+3.4 \mathrm{~L}, p=0.01)$. This suggests restoration of sustainable hemodynamics and resolution of ongoing capillary leak. This may be a good indicator for potential survivability, although it cannot unfortunately be predicted when first implanting ECMO. A strong focus on diuresis is a critical component to ECMO patient survival.

There were similar occurrences of bleeding events between patients that survived and those that did not survive, with a lower number of units of blood needed for resuscitation, for those patients that did survive (mean units of PRBCs for survivors was 2.7 units a day, compared to 4.7 units of PRBCs a day in non-survivors, $p=0.02$ ). Our patients required similar rates of red blood cell transfusions compared to prior studies [15-17]. Directed resuscitation with appropriate blood product use improves survival, but to our knowledge there have been no other studies measuring volume status and blood product usage $[3,10,18]$. Our study unmasks this potential link, therefore we suggest, appropriate diuresis and directed resuscitation suggest an important therapy to bridge a distressed myocardium and may aid the efforts of ECMO support.

Survival for V-A ECMO was $35 \%$ compared to $60 \%$ in $\mathrm{V}-\mathrm{V}$ ECMO. Even though this was found insignificant, it is unsurprising. Patients with two rather than one organ failing are more likely to have poor outcomes.

Cardiac reoperations were associated with worse survival outcomes while patients were on ECMO. Kidney failure, DVT/PE, ischemic limb, sepsis and intracerebral hemorrhage/anoxic brain injury had no significant mortality detriment, however there is a trend toward worse survival, with these mal-occurrences. Overall, only 3 patients had a DVT/PE. One thought for our overall low $\mathrm{DVT} / \mathrm{PE}$ rate is our patients placed on VA ECMO either had prophylactic superficial femoral artery catheter or had regional $\mathrm{SO}^{2}$ monitors on both feet showing good perfusion in the ipsilateral foot of the ECMO arterial side. We believe that in a larger sample size these values would become significant because we know they are significant in heart surgery patients who do not need ECMO.

We believe ECMO is a method for cardiopulmonary support to allow the body time to heal and to support end organ perfusion, as a bridge to recovery or to a more permanent treatment solution such as transplant or LVAD.
In our patients, a bridge-to-recovery approach was anticipated in the vast majority of the patients despite this cohort showing varying degrees of chronic heart failure pre-operatively. It has been shown that the potential for recovery may be limited in patients with underlying chronic heart failure and combined with advanced age, ECMO may be a futile intervention in the end. In resuscitated patients, a bridge-to-decision strategy for ECMO's use may be employed to help manage subsequent therapy such as LVAD surgery, implantable cardioverter defibrillator (ICD) implantation, and OHT. If the patient leaves the hospital with their own heart, it is important to avoid LV distention. Consequently, on ECMO support it is our recommendation to ensure adequate off-loading of the left ventricle (usually with an LV vent or Impella) and to minimize inopressor use to reduce myocardial oxygen demand and allow for myocardial recovery. It is unclear if this off-loading should be done for all patients preemptively when placed on ECMO support or if only after cardiac ECHO evaluation suggests that the LV is distended. We generally employee off-loading when it is indicated by $\mathrm{ECHO}$, but at this point we do not have any standard policy. Going forward this would be an interesting topic of research and standardization. In our 23 patients who survived two patients left with LVADs and 6 patients left with OHTs, 8 patients had some kind of assistance or another heart placed. More study is needed in this regard to help streamline the post-operative resuscitative course of patients who require post cardiotomy mechanical support.

If a patient's heart is failing there are options, one of which is ECMO. While these patients have drastically worse outcome than if they did not need ECMO, we think that it may allow us to salvage patients who are unsavable.

\section{Conclusion}

ECMO is a viable rescue strategy for cardiac surgery patients with a $40 \%$ survival to discharge rate. Careful attention to volume management and blood transfusion are important markers for potential survival.

\footnotetext{
Abbreviations

ECMO: Extracorporeal membrane oxygenation; CABG: Coronary artery bypass grafting; LVAD: Left ventricular assistant device; VA: Venous-arterial; VV: Venousvenous; VAV: Veno-arterio-venous; IABP: Intra-aortic ballon pump; CAD: Coronary artery disease; MI: Myocardial infarction; COPD: Chronic obstructive pulmonary disease; CHF: Congestive heart failure; PVD: Peripheral vascular disease; EF: Ejection fraction; DVT/PE: Deep venous thromboembolism/ pulmonary embolus; ICU: Intensive care unit; $\mathrm{OHT}$ : Open heart transplant; eCPR: ECMO cardiopulomary resuscitation; PRBCs: Packed red blood cells; ICD: Implantable cardioverter defibrillator.
}

Acknowledgements

Not applicable. 


\section{Authors' contributions}

JB finalized results, researched and primarily wrote the paper, AT designed the study, JY/MA/DP collected data, performed statistics and reviewed manuscript, JG/JG/DU designed study and reviewed manuscript. All authors read and approved the final manuscript.

\section{Funding}

We obtained no funding for the project.

\section{Availability of data and materials}

The datasets generated and/or analysed during the current study are not publicly available due to patient personal information included in the primary data set, but are available from the corresponding author on reasonable request.

\section{Declarations}

Ethics approval and consent to participate

We obtained approval from Hartford Hospital IRB (HHC-2019-0189).

\section{Consent for publication}

Not applicable.

\section{Competing interests}

The authors declare that they have no competing interests.

\section{Author details}

${ }^{1}$ Department of General Surgery, University of Connecticut, 263 Farmington Ave, Farmington, CT 06030, USA. ${ }^{2}$ Division of Trauma and Acute Care Surgery, Hartford Healthcare, Hartford, USA. ${ }^{3}$ Department of Cardiology and Mechanical Circulatory Support, Hartford Healthcare, Hartford, USA. ${ }^{4}$ Division of Cardiac Surgery, Hartford Healthcare, Hartford, USA

Received: 2 July 2021 Accepted: 29 August 2021

Published online: 19 September 2021

\section{References}

1. Baran DA. Extracorporeal membrane oxygenation (ECMO) and the critical cardiac patient. Curr Transplant Rep. 2017;4:218-25.

2. Grunau B, Carrier S, Bashir J, Dick W, Harris L, Boone H, et al. A comprehensive regional clinical and educational ECPR protocol decreases time to ECMO in patients with refractory out-of-hospital cardiac arrest. CJEM. 2017;19:424-33.

3. Hajiyev V, Erkenov T, Smechowski A, Soerren J, Frtizsche D. Follow-up on ECMO after cardiac surgery: how can we evaluate therapy? Heart Surg Forum. 2019:22:E011-E14.

4. Chonde M, Sappington P, Kormos R, Althouse A, Boujoukos A. The Use of ECMO for the treatment of refractory cardiac arrest or postarrest cardiogenic shock following in-hospital cardiac arrest: a 10-year experience. J Intensive Care Med. 2019;34:615-21.

5. Sun T, Guy A, Sidhu A, Finlayson G, Grunau B, Ding L, et al. Veno-arterial extracorporeal membrane oxygenation (VA-ECMO) for emergency cardiac support. J Crit Care. 2018:44:31-8.

6. Stub D, Bernard S, Pellegrino V, Smith K, Walker T, Sheldrake J, et al. Refractory cardiac arrest treated with mechanical CPR, hypothermia, ECMO and early reperfusion (the CHEER trial). Resuscitation. 2015;86:88-94.
7. Diez-Villanueva P, Sousa I, Nunez A, Diez F, Elzaga J, Fernandez-Aviles F. Early treatment of refractory cardiogenic shock with percutaneous venoarterial ECMO implanted in the cardiac catheterization laboratory. Rev Esp Cardiol (Engl Ed). 2014;67:1059-61.

8. Bigdeli AK, Deutsch MA, Beiras-Fernandez A, Michael S, Kaczmarek I, Schmitz C, Sodian R. ECMO after prolonged cardiopulmonary resuscitation as a successful bridge to immediate cardiac retransplant in a 6-yearold girl. Exp Clin Transplant. 2012;10:186-9.

9. Vardi A, Abed M, Mishali D, Levin I, Paret G, Barzilay Z. ECMO---extra corporeal membrane oxygenation as a bridge to recovery, following surgery for complex congenital cardiac anomalies, in children and neonates. Harefuah 2003;142:583-7, 648, 7.

10. Broome M, Donker DW. Individualized real-time clinical decision support to monitor cardiac loading during venoarterial ECMO. J Transl Med. 2016;14:4.

11. Garcia-Carreno J, Sousa-Casasnovas I, Devesa-Cordero C, Gutierrz-Ibanes E, Fernandez-Aviles F, Martinez-Selles M. Cardiopulmonary resuscitation with percutaneous ECMO in refractory in-hospital cardiac arrest: a singlecenter experience. Rev Esp Cardiol (Engl Ed). 2019;72:880-2.

12. Kacer J, Lindovska M, Surovcik R, Netuka I, Mlejnsky F, Grus T, et al. Refractory cardiogenic shock due to extensive anterior STEMI with covered left ventricular free wall rupture treated with awake VA-ECMO and LVAD as a double bridge to heart transplantation-collaboration of three cardiac centres. Biomed Pap Med Fac Univ Palacky Olomouc Czech Repub. 2015:159:681-7.

13. Adamson RM, Dembitsky WP, Daily PO, Moreno-Cabral R, Copeland J, Smith R. Immediate cardiac allograft failure. ECMO versus total artificial heart support. ASAIO J. 1996:42:314-6.

14. Hsu PS, Chen JL, Hong GJ, Tsai Y, Lin C, Lee C, et al. Extracorporeal membrane oxygenation for refractory cardiogenic shock after cardiac surgery: predictors of early mortality and outcome from 51 adult patients. Eur J Cardiothorac Surg. 2010;37(2):328-33. https://doi.org/10.1016/j.ejcts. 2009.07.033.

15. Rubino A, Diego C, Stanszus D, Valchanov K, Jenkins D, Sertic F, et al. Central veno-arterial extracorporeal membrane oxygenation (C-VA-ECMO) after cardiothoracic surgery: a single-center experience. J Cardiothorac Vasc Anesth. 2018;32(3):1169-74. https://doi.org/10.1053/j.jvca.2017.12. 003.

16. Biancari F, Perrotti A, Dalen M, Guerrieri M, Fiore A, Reichart D, et al. Metaanalysis of the outcome after postcardiotomy venoarterial extracorporeal membrane oxygenation in adult patients. J Cardiothorac Vasc Anesth. 2018;32(3):1175-82. https://doi.org/10.1053/j.jvca.2017.08.048.

17. Biancari F, Dalen M, Fiore A, Ruggiri V, Saeed D, Jonsson K, et al. Multicenter study on postcardiotomy venoarterial. J Thorac Cardiovasc Surg. 2020;159(5):1844-1854.e6. https://doi.org/10.1016/j.jtcvs.2019.06.039.

18. Dalle Ave AL, Shaw DM, Gardiner D. Extracorporeal membrane oxygenation (ECMO) assisted cardiopulmonary resuscitation or uncontrolled donation after the circulatory determination of death following out-ofhospital refractory cardiac arrest-an ethical analysis of an unresolved clinical dilemma. Resuscitation. 2016;108:87-94.

\section{Publisher's Note}

Springer Nature remains neutral with regard to jurisdictional claims in published maps and institutional affiliations. 